\title{
Application of anthropometric methods in the nursing process of nursing research
}

\author{
Aplicação de métodos antropométricos no processo de enfermagem de pesquisa em enfermagem \\ Aplicación de métodos antropométricos en el proceso de enfermería de la investigación enfermera
}

Silvia Domínguez Fernández'

ORCID: 0000-0002-6223-4051

Sonsoles García León" ORCID: 0000-0001-5902-3547

María Julia Ajejas Bazán' ORCID: 0000-0001-5343-3377

José Luis Pacheco del Cerro' ORCID: 0000-0001-6335-9086

Cristina Maria Alves Marques-Vieira"'I ORCID: 0000-0002-4409-7911

Francisco Javier Pérez Rivas' ORCID: 0000-0003-4294-8417

'Universidad Complutense de Madrid. Madrid, España. "Hospital General Universitario Gregorio Marañón. Madrid, España.

"'Universidade Católica Portuguesa. Lisboa, Portugal.

How to cite this article: Fernández SD, León SG, Bazán MJA, Cerro JLP Marques-Vieira CMA, Rivas FJP. Application of anthropometric methods in the nursing process of nursing research.

Rev Bras Enferm. 2020;73(Suppl 6):e20190604. doi: http://dx.doi.org/10.1590/0034-7167-2019-0604

Corresponding author: Silvia Domínguez Fernández E-mail: sildom01@ucm.es

EDITOR IN CHIEF: Antonio José de Almeida Filho ASSOCIATE EDITOR: Marcos Brandão

\section{ABSTRACT}

Objectives: to identify anthropometric techniques and measurements related to nursing diagnoses and interventions, included in the NANDA International (NANDA-I) and Nursing Interventions Classification (NIC), respectively. Methods: descriptive study of the revision of the NANDA-I and NIC taxonomies by professors of Nursing of the Universidad Complutense de Madrid. Results: A total of 24 nursing diagnoses, 20 nursing interventions and 71 nursing activities were identified that require the use of anthropometric measurements. Conclusions: anthropometric measurements are necessary in the conduct of numerous nursing diagnoses and nursing interventions that are commonly used in healthcare practice. It would be useful to systematize the use of anthropometric techniques in the application of the nursing process and to standardize this training process.

Descriptors: Anthropometry, Nursing Process, Nursing Diagnosis, Nursing Research, Reference Standards.

\section{RESUMO}

Objetivos: Identificar técnicas e medidas antropométricas relacionadas com diagnósticos e intervenções de enfermagem, incluídas na NANDA-Internacional (NANDA-I) e na Nursing Interventions Classification (NIC) respectivamente. Métodos: estudo descritivo. Revisão das taxonomias NANDA-I e NIC por Professores de Enfermagem da Universidade Complutense de Madrid. Resultados: foram identificados 24 diagnósticos de enfermagem, 20 intervenções de enfermagem e 71 atividades de enfermagem que exigem o uso de medidas antropométricas. Conclusões: as medidas antropométricas são necessárias na realização de diversos diagnósticos de enfermagem e intervenções de enfermagem comummente utilizadas na prática assistencial. Seria útil sistematizar o uso de técnicas antropométricas na aplicação do processo de enfermagem e padronizar o processo de formação.

Descritores: Antropometria, Processo de Enfermagem, Diagnóstico de Enfermagem, Pesquisa em Enfermagem, Padrões de Referência.

\section{RESUMEN}

Objetivos: identificar técnicas y medidas antropométricas relacionadas con diagnósticos e intervenciones de enfermería, incluidas en NANDA-International (NANDA-I) y Clasificación de Intervenciones de Enfermería (NIC), respectivamente. Métodoss: estudio descriptivo. Revisión de las taxonomías NANDA-I y NIC por profesores de Enfermería de la Universidad Complutense de Madrid. Resultados: se identificaron 24 diagnósticos de enfermería, 20 intervenciones de enfermería y 71 actividades de enfermería que requerían el uso de mediciones antropométricas. Conclusiones: las mediciones antropométricas son necesarias para establecer numerosos diagnósticos e intervenciones de enfermería, comúnmente utilizados en la práctica asistencial. Sería útil sistematizar el uso de técnicas antropométricas en la aplicación del proceso de enfermería y estandarizar el proceso de capacitación de las enfermeras.

Descriptores: Antropometría, Proceso de Enfermería, Diagnóstico de Enfermería, Investigación en Enfermería, Estándares de Referencia.

Submission: 08-11-2019 Approval: 06-03-2020 


\section{INTRODUCTION}

The nursing process (or nursing methodology) consists of the application of the scientific method to the clinical practice of nursing. Alfaro Lefevre defines the nursing process as the systematic, humanistic and rational method for organizing and providing nursing care ${ }^{(1)}$.

This process is developed in five sequential phases (assessment, nursing diagnosis, planning, execution and evaluation), among which the nursing diagnosis stands out, which many authors consider the axis of the care plan ${ }^{(2)}$. NANDA International (NANDA-I) defines the nursing diagnosis as a clinical judgment about a human response to health conditions/life processes, or a vulnerability to that response, by an individual, family, group or community ${ }^{(3)}$. A nursing diagnosis provides the basis for the selection of nursing interventions to achieve results for which the nurse is responsible ${ }^{(3)}$.

The use of the nursing process in general, and of the nursing diagnosis in particular, allows for differentiating the specific contributions of nurses from other professional actions ${ }^{(4)}$.

NANDA- $\left.\right|^{(3)}$, the Nursing Interventions Classification $(\mathrm{NIC})^{(5)}$ and the Nursing Outcomes Classification (NOC)( ${ }^{(6)}$ are standardized classifications of nursing diagnoses, nursing interventions and outcome criteria specific to nursing practice, respectively. The implementation of the three classification NNN interrelationships (nursing diagnosis, intervention and outcome classifications) in the electronic nursing documentation system helps nurses use the nursing process, contributes to improving diagnostic accuracy and facilitates the evaluation of the outcomes derived from the nursing intervention ${ }^{(7)}$.

Taxonomy II of NANDA-I provides a structure that classifies the 235 nursing diagnoses into seven domains and 47 classes $^{(3)}$.

The nursing diagnoses approved by NANDA-I contain, in addition to the diagnostic label and its definition for all cases, the related factors and defining characteristics in the actual nursing diagnoses and the risk factors in the risk diagnoses.

The NIC (5) also consists of a structure of domains, classes and interventions. Each of the 550 interventions included in the classification has a title, definition, list of activities and bibliographic references. This structure favors the use of a standardized language in the process of making clinical and therapeutic decisions and facilitates the support of the process of documentation of professional practice ${ }^{(8)}$.

Anthropometry can be defined as the set of techniques that allows the characterization of human morphology, as well as body diversity, due to sexual, age or ethnic group differences. It also assesses the influence of the environment on body size and shape, including nutrition, health status and social or cultural customs ${ }^{(9)}$.

Specifically, the anthropometry of health analyzes the relationship between the morphological variability of the human being and the predisposition to contract diseases or suffer them directly. The most obvious example is the use of the waist-to-height ratio to determine cardiovascular risk.

The provision of nursing care and, by extension, the use of the nursing process requires numerous anthropometric techniques: direct measurements, such as weight, height and body fat percentage; and indirect measurements, of which the most commonly used is the body mass index (BMI). In addition, other indices, such as the waistheight index, are used to measure robustness and proportionality.

Neither the anthropometric techniques identified in the different stages of the nursing process nor the level of use of these techniques have been determined. It would be very useful to systematize their use and to identify in each case the most appropriate measure or anthropometric index for the nursing diagnosis or the most appropriate nursing intervention. In addition, objective data provided by anthropometric techniques may contribute to improving the validity and safety of nurses' determinations increasing diagnostic accuracy and facilitating the monitoring and evaluation of the whole nursing process.

\section{OBJECTIVES}

To identify anthropometric techniques and measurements related to nursing diagnoses and interventions, included in the NANDA-I and NIC classifications, respectively.

\section{METHODS}

\section{Ethical aspects}

For the development of the research was considered an ethical structure to provide the information clearly and truthfully. Copyright of taxonomies NANDA and NIC taxonomies has been respected by incorporating the corresponding bibliographic references. None of the authors present a conflict of interest in the elaboration of the article.

\section{Design, period and place of study}

Descriptive study, carried out from January 2017 to January 2018 by professors of the Facultad de Enfermeria, Universidad Complutense de Madrid and consisted of the review of the NANDA-I 2015-2017 ${ }^{(3)}$ and NIC 2012 $2^{(5)}$ taxonomies.

The reference of the EQUATOR network used was STROBE for Observational studies.

\section{Population or sample; criteria of inclusion and exclusion}

The review of the NANDA-I 2015-2017(3) and NIC $2012^{(5)}$ taxonomies, taking into account the following review criteria: All nursing diagnoses were analyzed by domains and classes, selecting those in which, for the identification of risk factors, related factors or defining characteristics, it is necessary to use anthropometric techniques. Likewise, the interventions and activities of the NIC classification related to the application of anthropometric techniques were reviewed.

\section{Study protocol}

The procedure to select the anthropometric techniques related to the nursing diagnoses and interventions was carried out in several phases:

- Identification by all members of the work team of the anthropometric techniques used in the provision of nursing care. The techniques required were not the same for nursing diagnoses as for interventions and activities. This was done using a nominal group technique ${ }^{(10-11)}$. Raising the initial question (what anthropometric techniques are required for nursing care?) each member of the research group (authors 
of the article) wrote their proposal individually. The following step was compiled the proposals and displayed to the entire group. The various proposals were clarified and discussed by all the panelists. Finally, the consensus of the anthropometric techniques was established when at least $75 \%$ of the panelists expressed their agreement with the proposal.

- Comprehensive and independent review of the NANDA-I and NIC taxonomies by two members of the research team, establishing relationships with anthropometric techniques.

- A third member reviewed the existing discrepancies, making an integrative proposal that was accepted by the entire team.

\section{Analysis of results and statistics}

The data collected were organized into spreadsheet using Microsoft Office Excel 2010. In-depth analysis of the NANDA-I nursing diagnosis classifications and NIC classification to identify those that require different anthropometric measurements. Descriptive statistics was used to describe and summarize the results found.

\section{RESULTS}

After an in-depth analysis of the NANDA-I nursing diagnosis classifications, a total of 24 nursing diagnoses (10.2\%) (Chart 1) that require different anthropometric measurements were identified, either for the identification of related factors or risk factors or for the validation of the defining characteristics. Between two and seven anthropometric measurements are used per nursing diagnosis. The nursing diagnoses susceptible to the use of more anthropometric techniques were those related to mobility (Chart 1).

The anthropometric measurements that are required in the nursing diagnostic stage are weight, $\mathrm{BMI}$, mid-upper arm circumference/arm circumference (MUAC/AC), body fat percentage, height-weight curves, height, waist circumference and muscle mass.

In relation to the nursing interventions of the NIC classification, a total of 20 nursing interventions (3.6\%) were identified (Chart 2) in which different anthropometric measurements are required for its realization. In these interventions, between two and three measurements are used per intervention. The interventions requiring more anthropometric techniques were weight management and nutrition therapy, which both need three anthropometric measurements (Chart 2).

The anthropometric measurements that are required for performing the different nursing interventions are weight, ideal weight, body fat percentage, BMI, waist circumference and triceps skin fold thickness/MUAC.

A total of 71 nursing activities also require an anthropometric technique or measurement (Chart 3).

Chart 1 - NANDA-International that use anthropometric measurements, Madrid, Spain, 2018

\begin{tabular}{|c|c|c|c|c|c|c|c|c|c|c|}
\hline DOMAIN & CLASS & $\begin{array}{l}\text { NURSING } \\
\text { DIAGNOSIS }\end{array}$ & WEIGHT & BMI & MUAC/AC & $\begin{array}{l}\text { PERCENT } \\
\text { BODY FAT }\end{array}$ & $\begin{array}{l}\text { HEIGHT } \\
\text { CURVES }\end{array}$ & HEIGHT & $\begin{array}{c}\text { WAIST } \\
\text { CIRCUMFERENCE }\end{array}$ & $\begin{array}{c}\text { MUSCLE } \\
\text { MASS }\end{array}$ \\
\hline \multirow{3}{*}{$\begin{array}{l}\text { DOMAIN 1: } \\
\text { Health } \\
\text { promotion }\end{array}$} & $\begin{array}{l}\text { CLASS 1: } \\
\text { HEALTH AWARENESS }\end{array}$ & $\begin{array}{l}\text { [00168] Sedentary } \\
\text { lifestyle }\end{array}$ & $x$ & $x$ & & $x$ & & & & \\
\hline & \multirow{2}{*}{$\begin{array}{l}\text { CLASS 2: } \\
\text { HEALTH MANAGEMENT }\end{array}$} & $\begin{array}{l}\text { [00231] Risk } \\
\text { for frail elderly } \\
\text { syndrome }\end{array}$ & $X$ & $x$ & $X$ & $X$ & & $x$ & & $X$ \\
\hline & & $\begin{array}{l}\text { [00257] Frail } \\
\text { elderly syndrome }\end{array}$ & $x$ & $x$ & $X$ & $x$ & & $x$ & & $X$ \\
\hline \multirow{4}{*}{$\begin{array}{l}\text { DOMAIN 2: } \\
\text { Nutrition }\end{array}$} & \multirow{4}{*}{$\begin{array}{l}\text { CLASS 1: } \\
\text { INGESTION }\end{array}$} & $\begin{array}{l}\text { [00002] } \\
\text { Imbalanced } \\
\text { nutrition: less than } \\
\text { body requirement }\end{array}$ & $x$ & $x$ & & & & $X$ & $x$ & \\
\hline & & [00232] Obesity & $x$ & $x$ & & & & $x$ & $x$ & \\
\hline & & $\begin{array}{l}\text { [00233] } \\
\text { Overweight }\end{array}$ & $X$ & $x$ & & & & $x$ & $x$ & \\
\hline & & $\begin{array}{l}\text { [00234] Risk for } \\
\text { overweight }\end{array}$ & $X$ & $x$ & & & & $x$ & $X$ & \\
\hline \multirow{6}{*}{$\begin{array}{l}\text { DOMAIN 4: } \\
\text { Activity/Rest }\end{array}$} & \multirow{6}{*}{$\begin{array}{l}\text { CLASS 2: } \\
\text { ACTIVITY/EXERCISE }\end{array}$} & $\begin{array}{l}\text { [00085] Impaired } \\
\text { physical mobility }\end{array}$ & $X$ & $x$ & $x$ & $x$ & & $X$ & & $x$ \\
\hline & & $\begin{array}{l}\text { [00088] Impaired } \\
\text { walking }\end{array}$ & $X$ & $x$ & $x$ & $x$ & & $X$ & & $x$ \\
\hline & & $\begin{array}{l}\text { [00089] Impaired } \\
\text { wheelchair } \\
\text { mobility }\end{array}$ & $X$ & $x$ & $X$ & $X$ & & $X$ & & $X$ \\
\hline & & $\begin{array}{l}\text { [00090] Impaired } \\
\text { transfer ability }\end{array}$ & $x$ & $x$ & $x$ & $x$ & & $X$ & & $X$ \\
\hline & & $\begin{array}{l}\text { [00091] Impaired } \\
\text { bed mobility }\end{array}$ & $x$ & $x$ & $x$ & $x$ & & $x$ & & $X$ \\
\hline & & $\begin{array}{l}\text { [00237] Impaired } \\
\text { sitting }\end{array}$ & $x$ & $X$ & $X$ & $X$ & & $x$ & & $X$ \\
\hline
\end{tabular}




\begin{tabular}{|c|c|c|c|c|c|c|c|c|c|c|}
\hline DOMAIN & CLASS & $\begin{array}{l}\text { NURSING } \\
\text { DIAGNOSIS }\end{array}$ & WEIGHT & BMI & MUAC/AC & $\begin{array}{l}\text { PERCENT } \\
\text { BODY FAT } \\
\end{array}$ & $\begin{array}{l}\text { HEIGHT } \\
\text { CURVES } \\
\end{array}$ & HEIGHT & $\begin{array}{c}\text { WAIST } \\
\text { CIRCUMFERENCE } \\
\end{array}$ & $\begin{array}{c}\text { MUSCLE } \\
\text { MASS }\end{array}$ \\
\hline \multirow{5}{*}{$\begin{array}{l}\text { DOMAIN 4: } \\
\text { Activity/Rest }\end{array}$} & \multirow{5}{*}{$\begin{array}{l}\text { CLASS 2: } \\
\text { ACTIVITY/EXERCISE }\end{array}$} & $\begin{array}{l}\text { [00238] Impaired } \\
\text { standing }\end{array}$ & $x$ & $x$ & $x$ & $x$ & & $x$ & & $x$ \\
\hline & & [00093] Fatigue & $x$ & $x$ & $x$ & $x$ & & $x$ & & $x$ \\
\hline & & $\begin{array}{l}\text { [00092] Activity } \\
\text { intolerance }\end{array}$ & $x$ & $x$ & $x$ & $x$ & & $x$ & & $x$ \\
\hline & & $\begin{array}{l}\text { [00094] Risk } \\
\text { for activity } \\
\text { intolerance }\end{array}$ & $x$ & $x$ & $x$ & $x$ & & $x$ & & $x$ \\
\hline & & $\begin{array}{l}\text { [00102] Feeding } \\
\text { self-care deficit }\end{array}$ & $x$ & $x$ & & & & $x$ & $x$ & \\
\hline $\begin{array}{l}\text { DOMAIN 6: } \\
\text { Self-perception }\end{array}$ & $\begin{array}{l}\text { CLASS 3: } \\
\text { BODY IMAGE }\end{array}$ & $\begin{array}{l}\text { [00118] Disturbed } \\
\text { body image }\end{array}$ & $x$ & $x$ & & $x$ & & $x$ & & $x$ \\
\hline \multirow{4}{*}{$\begin{array}{l}\text { DOMAIN 11: } \\
\text { Safety/ } \\
\text { Protection }\end{array}$} & \multirow{3}{*}{$\begin{array}{l}\text { CLASS 2: } \\
\text { PHYSICAL INJURY }\end{array}$} & $\begin{array}{l}\text { [00035] Risk for } \\
\text { injury }\end{array}$ & $x$ & $x$ & $x$ & $x$ & & $x$ & & $x$ \\
\hline & & $\begin{array}{l}\text { [00155] Risk for } \\
\text { falls }\end{array}$ & $x$ & $x$ & $x$ & $x$ & & $x$ & & $x$ \\
\hline & & $\begin{array}{l}\text { [00249] Risk for } \\
\text { pressure ulcer }\end{array}$ & $x$ & $x$ & & & & $x$ & $x$ & \\
\hline & $\begin{array}{l}\text { CLASS 6: } \\
\text { THERMOREGULATION }\end{array}$ & $\begin{array}{l}{[00005] \text { Risk for }} \\
\text { imbalanced body } \\
\text { temperature }\end{array}$ & $x$ & $x$ & & & & $x$ & & \\
\hline $\begin{array}{l}\text { DOMAIN 13: } \\
\text { Growth/ } \\
\text { Development }\end{array}$ & $\begin{array}{l}\text { CLASS 1: } \\
\text { GROWTH }\end{array}$ & $\begin{array}{l}\text { [00113] Risk for } \\
\text { disproportionate } \\
\text { growth }\end{array}$ & & & & & $x$ & $x$ & & \\
\hline
\end{tabular}

Chart 2 - NIC that require an anthropometric technique or measurement to be performed, Madrid, Spain, 2018

\begin{tabular}{|c|c|c|c|c|c|c|c|c|}
\hline DOMAIN & CLASS & INTERVENTIONS & WEIGHT & BMI & $\begin{array}{c}\text { IDEAL } \\
\text { WEIGHT }\end{array}$ & $\begin{array}{l}\text { PERCENT } \\
\text { BODY FAT }\end{array}$ & $\begin{array}{c}\text { WAIST } \\
\text { CIRCUMFERENCE }\end{array}$ & $\begin{array}{c}\text { TRICEPS SKIN } \\
\text { FOLD /MID-UPPER } \\
\text { ARM MUSCLE } \\
\text { CIRCUMFERENCE }\end{array}$ \\
\hline \multirow{2}{*}{$\begin{array}{l}\text { DOMAIN 1: } \\
\text { Physiological: } \\
\text { Basic }\end{array}$} & \multirow{2}{*}{$\begin{array}{l}\text { CLASS A: } \\
\text { Activity and Exercise } \\
\text { Management }\end{array}$} & $\begin{array}{l}\text { [0226] Exercise therapy: } \\
\text { muscle control }\end{array}$ & & $\mathrm{X}$ & & & $X$ & \\
\hline & & $\begin{array}{l}\text { [0180] Energy } \\
\text { management }\end{array}$ & $x$ & & & & & \\
\hline \multirow{10}{*}{$\begin{array}{l}\text { DOMAIN 1: } \\
\text { Physiological: } \\
\text { Basic }\end{array}$} & \multirow{10}{*}{$\begin{array}{l}\text { CLASS D: } \\
\text { Nutrition Support }\end{array}$} & [1050] Feeding & $x$ & & & & & \\
\hline & & $\begin{array}{l}\text { [5246] Nutritional } \\
\text { counseling }\end{array}$ & $\mathrm{x}$ & & & & & \\
\hline & & $\begin{array}{l}\text { [1803] Self-care } \\
\text { assistance: feeding }\end{array}$ & $\mathrm{x}$ & & & & & \\
\hline & & $\begin{array}{l}\text { [1280] Weight reduction } \\
\text { assistance }\end{array}$ & $\mathrm{x}$ & & & $\mathrm{x}$ & & \\
\hline & & $\begin{array}{l}\text { [1240] Weight gain } \\
\text { assistance }\end{array}$ & $X$ & & & & & \\
\hline & & $\begin{array}{l}\text { [1100] Nutrition } \\
\text { management }\end{array}$ & $\mathrm{x}$ & & & & & \\
\hline & & $\begin{array}{l}\text { [1030] Eating disorders } \\
\text { management }\end{array}$ & $\mathrm{x}$ & & & & & \\
\hline & & $\begin{array}{l}\text { [1260] Weight } \\
\text { management }\end{array}$ & $\mathrm{x}$ & & $\mathrm{x}$ & $\mathrm{x}$ & & \\
\hline & & [1120] Nutrition therapy & $x$ & $x$ & & $x$ & & \\
\hline & & $\begin{array}{l}{[1160] \text { Nutritional }} \\
\text { monitoring }\end{array}$ & & & & & & $x$ \\
\hline $\begin{array}{l}\text { DOMAIN 2: } \\
\text { Physiological: } \\
\text { Complex }\end{array}$ & $\begin{array}{l}\text { CLASS L: } \\
\text { Skin/Wound } \\
\text { Management }\end{array}$ & [3520] Pressure ulcer care & $\mathrm{x}$ & & & & & \\
\hline
\end{tabular}


Chart 2 (concluded)

\begin{tabular}{|c|c|c|c|c|c|c|c|c|}
\hline DOMAIN & CLASS & INTERVENTIONS & WEIGHT & BMI & $\begin{array}{c}\text { IDEAL } \\
\text { WEIGHT }\end{array}$ & $\begin{array}{l}\text { PERCENT } \\
\text { BODY FAT }\end{array}$ & $\begin{array}{c}\text { WAIST } \\
\text { CIRCUMFERENCE }\end{array}$ & $\begin{array}{l}\text { TRICEPS SKIN } \\
\text { FOLD /MID-UPPER } \\
\text { ARM MUSCLE } \\
\text { CIRCUMFERENCE }\end{array}$ \\
\hline $\begin{array}{l}\text { DOMAIN 2: } \\
\text { Physiological: } \\
\text { Complex }\end{array}$ & $\begin{array}{l}\text { CLASS N: } \\
\text { Tissue Perfusion } \\
\text { Management }\end{array}$ & [4120] Fluid management & $\mathrm{x}$ & & & & & \\
\hline $\begin{array}{l}\text { DOMAIN 2: } \\
\text { Physiological: } \\
\text { Complex }\end{array}$ & $\begin{array}{l}\text { CLASS G: } \\
\text { Electrolyte and Acid- } \\
\text { Base Management }\end{array}$ & $\begin{array}{l}\text { [2080] Fluid/electrolyte } \\
\text { management }\end{array}$ & $\mathrm{x}$ & & & & & \\
\hline $\begin{array}{l}\text { DOMAIN 2: } \\
\text { Physiological: } \\
\text { Complex }\end{array}$ & $\begin{array}{l}\text { CLASS N: } \\
\text { Tissue Perfusion } \\
\text { Management } \\
\end{array}$ & [4040] Cardiac care & $\mathrm{X}$ & & & & & \\
\hline $\begin{array}{l}\text { DOMAIN 2: } \\
\text { Physiological: } \\
\text { Complex }\end{array}$ & $\begin{array}{l}\text { CLASS N: } \\
\text { Tissue Perfusion } \\
\text { Management } \\
\end{array}$ & $\begin{array}{l}\text { [4250] Shock } \\
\text { management }\end{array}$ & $\mathrm{x}$ & & & & & \\
\hline $\begin{array}{l}\text { DOMAIN } 3 . \\
\text { Behavioral }\end{array}$ & $\begin{array}{l}\text { CLASS R: } \\
\text { Coping Assistance }\end{array}$ & $\begin{array}{l}\text { [5220] Body image } \\
\text { enhancement }\end{array}$ & $\mathrm{x}$ & $\mathrm{x}$ & & & & \\
\hline $\begin{array}{l}\text { DOMAIN 2: } \\
\text { Physiological: } \\
\text { Complex }\end{array}$ & $\begin{array}{l}\text { CLASS N: } \\
\text { Tissue Perfusion } \\
\text { Management }\end{array}$ & [4130] Fluid monitoring & $x$ & & & & & \\
\hline $\begin{array}{l}\text { DOMAIN 2: } \\
\text { Physiological: } \\
\text { Complex }\end{array}$ & $\begin{array}{l}\text { CLASS N: } \\
\text { Tissue Perfusion } \\
\text { Management }\end{array}$ & [4260] Shock prevention & $\mathrm{x}$ & & & & & \\
\hline
\end{tabular}

Chart 3 - Nursing activities identified where anthropometric measurements are made, Madrid, Spain, 2018

\begin{tabular}{|c|c|c|c|}
\hline & & INTERVENCIONES & ACTIVIDADES \\
\hline \multirow{2}{*}{$\begin{array}{l}\text { DOMAIN 1: } \\
\text { Physiological: } \\
\text { Basic }\end{array}$} & \multirow{2}{*}{$\begin{array}{l}\text { CLASS D: } \\
\text { Nutrition Support }\end{array}$} & \multirow{2}{*}{ Feeding } & 1. Identify the prescribed diet. \\
\hline & & & 2. Record the intake, if appropriate. \\
\hline \multirow{5}{*}{$\begin{array}{l}\text { DOMAIN 1: } \\
\text { Physiological: } \\
\text { Basic }\end{array}$} & \multirow{5}{*}{$\begin{array}{l}\text { CLASS D: } \\
\text { Nutrition Support }\end{array}$} & \multirow{5}{*}{$\begin{array}{l}\text { Nutritional } \\
\text { counseling }\end{array}$} & 1. Determine the patient's intake and eating habits. \\
\hline & & & 2. Set realistic mid- and short-term goals for the change in nutritional status. \\
\hline & & & 3. Use accepted dietary standards to help the patient assess the appropriate dietary intake. \\
\hline & & & 4. Help the patient record what he usually eats in a 24 -hour period. \\
\hline & & & $\begin{array}{l}\text { 5. Review with the patient the measurement of fluid intake and elimination, hemoglobin values, } \\
\text { blood pressure readings or weight gain and loss, if applicable. }\end{array}$ \\
\hline $\begin{array}{l}\text { DOMAIN 1: } \\
\text { Physiological: } \\
\text { Basic } \\
\end{array}$ & $\begin{array}{l}\text { CLASS D: } \\
\text { Nutrition Support }\end{array}$ & \begin{tabular}{|l|} 
Self-care \\
assistance: \\
feeding \\
\end{tabular} & 1. Check the patient's weight, if applicable. \\
\hline \multirow{5}{*}{$\begin{array}{l}\text { DOMAIN 1: } \\
\text { Physiological: } \\
\text { Basic }\end{array}$} & \multirow{5}{*}{$\begin{array}{l}\text { CLASS D: } \\
\text { Nutrition Support }\end{array}$} & \multirow{5}{*}{$\begin{array}{l}\text { Weight reduction } \\
\text { assistance }\end{array}$} & 1. Determine the patient's desire and motivation to reduce weight or body fat. \\
\hline & & & 2. Determine with the patient the amount of weight loss desired. \\
\hline & & & 3. Set a weekly weight loss goal. \\
\hline & & & 4. Weigh the patient weekly. \\
\hline & & & $\begin{array}{l}\text { 5. Recommend the adoption of diets that lead to the achievement of long-term goals in } \\
\text { weight loss. }\end{array}$ \\
\hline \multirow{4}{*}{$\begin{array}{l}\text { DOMAIN 1: } \\
\text { Physiological: } \\
\text { Basic }\end{array}$} & \multirow{4}{*}{$\begin{array}{l}\text { CLASS D: } \\
\text { Nutrition Support }\end{array}$} & \multirow{4}{*}{$\begin{array}{l}\text { Weight gain } \\
\text { assistance }\end{array}$} & 1. Weigh the patient at the intervals determined, if applicable. \\
\hline & & & 2. Analyze the possible causes of low body weight. \\
\hline & & & 3. Periodically monitor the daily consumption of calories. \\
\hline & & & 4. Periodically check serum albumin, lymphocyte and electrolyte levels. \\
\hline $\begin{array}{l}\text { DOMAIN 1: } \\
\text { Physiological: } \\
\text { Basic } \\
\end{array}$ & $\begin{array}{l}\text { CLASS A: } \\
\text { Activity and Exercise } \\
\text { Management }\end{array}$ & $\begin{array}{l}\text { Energy } \\
\text { management }\end{array}$ & 1. Control the dietary intake to ensure sufficient energy resources. \\
\hline \multirow{7}{*}{$\begin{array}{l}\text { DOMAIN 1: } \\
\text { Physiological: } \\
\text { Basic }\end{array}$} & \multirow{7}{*}{$\begin{array}{l}\text { CLASS A: } \\
\text { Activity and Exercise } \\
\text { Management }\end{array}$} & \multirow{7}{*}{$\begin{array}{l}\text { Nutritional } \\
\text { management }\end{array}$} & 1. Determine the patient's nutritional status and their ability to meet nutritional needs. \\
\hline & & & 2. Instruct the patient about nutritional needs. \\
\hline & & & $\begin{array}{l}\text { 3. Help the patient determine the guidelines or food pyramids most appropriate to meet } \\
\text { nutritional needs and preferences. }\end{array}$ \\
\hline & & & 4. Adjust the diet as necessary. \\
\hline & & & 5. Monitor calories and food intake. \\
\hline & & & 6. Monitor weight loss and weight gain trends. \\
\hline & & & 7. Teach the patient to control calories and dietary intake. \\
\hline
\end{tabular}


Chart 3

\begin{tabular}{|c|c|c|c|}
\hline & & INTERVENCIONES & ACTIVIDADES \\
\hline \multirow{6}{*}{$\begin{array}{l}\text { DOMAIN 1: } \\
\text { Physiological: } \\
\text { Basic }\end{array}$} & \multirow{6}{*}{$\begin{array}{l}\text { CLASS D: } \\
\text { Nutrition Support }\end{array}$} & \multirow{6}{*}{$\begin{array}{l}\text { Eating disorders } \\
\text { management }\end{array}$} & 1. Establish the desired weight gain. \\
\hline & & & 2. Monitor the necessary physiological parameters (vital signs and electrolyte levels). \\
\hline & & & 3. Weigh daily (at the same time of day and after evacuation). \\
\hline & & & 4. Monitor the daily caloric intake of food. \\
\hline & & & 5. Control the patient's weight routinely. \\
\hline & & & 6. Determine the acceptable margin of weight variation relative to the reference margin. \\
\hline \multirow{3}{*}{$\begin{array}{l}\text { DOMAIN 1: } \\
\text { Physiological: } \\
\text { Basic }\end{array}$} & \multirow{3}{*}{$\begin{array}{l}\text { CLASS D: } \\
\text { Nutrition Support }\end{array}$} & \multirow{3}{*}{$\begin{array}{l}\text { Weight } \\
\text { management }\end{array}$} & 1. Determine the ideal body weight of the individual. \\
\hline & & & 2. Determine the ideal body fat percentage of the individual. \\
\hline & & & 3. Encourage the individual to record their weight weekly, if applicable. \\
\hline \multirow{6}{*}{$\begin{array}{l}\text { DOMAIN 1: } \\
\text { Physiological: } \\
\text { Basic }\end{array}$} & \multirow{6}{*}{$\begin{array}{l}\text { CLASS D: } \\
\text { Nutrition Support }\end{array}$} & \multirow{6}{*}{$\begin{array}{l}\text { Nutritional } \\
\text { monitoring }\end{array}$} & 1. Weigh the patient at the established intervals. \\
\hline & & & 2. Monitor the trends of weight loss and gain. \\
\hline & & & $\begin{array}{l}\text { 3. Monitor skin fold measurements: triceps skin fold thickness, mid-upper arm muscle } \\
\text { circumference and mid-upper arm circumference. }\end{array}$ \\
\hline & & & 4. Monitor levels of albumin, total protein, hemoglobin and hematocrit. \\
\hline & & & 5. Check growth and development. \\
\hline & & & 6. Control caloric and nutritional intake. \\
\hline \multirow{2}{*}{$\begin{array}{l}\text { DOMAIN 1: } \\
\text { Physiological: } \\
\text { Basic }\end{array}$} & \multirow{2}{*}{$\begin{array}{l}\text { CLASS A: } \\
\text { Activity and Exercise } \\
\text { Management }\end{array}$} & \multirow{2}{*}{$\begin{array}{l}\text { Exercise therapy: } \\
\text { muscle control }\end{array}$} & 1. Determine the accuracy of the body image. \\
\hline & & & 2. Reorient the patient regarding body awareness. \\
\hline \multirow{4}{*}{$\begin{array}{l}\text { DOMAIN 1: } \\
\text { Physiological: } \\
\text { Basic }\end{array}$} & \multirow{4}{*}{$\begin{array}{l}\text { CLASS D: } \\
\text { Nutrition Support }\end{array}$} & \multirow{4}{*}{ Nutrition therapy } & 1. Complete a nutritional assessment, if applicable. \\
\hline & & & 2. Control the ingested food/fluids and calculate the daily caloric intake, as appropriate. \\
\hline & & & 3. Control laboratory values, as appropriate. \\
\hline & & & 4. Check whether dietary guidelines meet daily dietary needs, if applicable. \\
\hline \multirow{3}{*}{$\begin{array}{l}\text { DOMAIN 2: } \\
\text { Physiological: } \\
\text { Complex }\end{array}$} & \multirow{3}{*}{$\begin{array}{l}\text { CLASS L: } \\
\text { Skin/Wound } \\
\text { Management }\end{array}$} & \multirow{3}{*}{$\begin{array}{l}\text { Pressure ulcer } \\
\text { care }\end{array}$} & 1. Ensure sufficient dietary intake. \\
\hline & & & 2. Control nutritional status. \\
\hline & & & 3. Verify sufficient caloric intake and high-quality protein intake. \\
\hline \multirow{4}{*}{$\begin{array}{l}\text { DOMAIN 2: } \\
\text { Physiological: } \\
\text { Complex }\end{array}$} & \multirow{4}{*}{$\begin{array}{l}\text { CLASS N: } \\
\text { Tissue Perfusion } \\
\text { Management }\end{array}$} & \multirow{4}{*}{$\begin{array}{l}\text { Fluid } \\
\text { management }\end{array}$} & 1. Weigh daily and control evolution. \\
\hline & & & 2. Check for patient weight changes before and after dialysis, if applicable. \\
\hline & & & 3. Control food/fluid intake and calculate caloric intake, as appropriate. \\
\hline & & & 4. Monitor the nutritional status. \\
\hline \multirow{3}{*}{$\begin{array}{l}\text { DOMAIN 2: } \\
\text { Physiological: } \\
\text { Complex }\end{array}$} & \multirow{3}{*}{$\begin{array}{l}\text { CLASS G: Electrolyte } \\
\text { and Acid-Base } \\
\text { Management }\end{array}$} & \multirow{3}{*}{$\begin{array}{l}\text { Fluid/electrolyte } \\
\text { management }\end{array}$} & 1. Weigh daily and assess the evolution. \\
\hline & & & 2. Keep accurate records of intake and output. \\
\hline & & & 3. Provide the appropriate prescribed diet to restore the specific fluid or electrolyte balance. \\
\hline \multirow{2}{*}{$\begin{array}{l}\text { DOMAIN 2: } \\
\text { Physiological: } \\
\text { Complex }\end{array}$} & CLASS N: & I Cardiaccar & $\begin{array}{l}\text { 1. Encourage the patient to maintain caloric intake at a level that allows achieving the desired } \\
\text { weight. }\end{array}$ \\
\hline & $\begin{array}{l}\text { IIssue Pertusion } \\
\text { Management }\end{array}$ & - Caralac care & $\begin{array}{l}\text { 2. Focus the assistance and therapeutic objectives to allow the patient to continue controlling } \\
\text { their weight, not smoking and to be as active as possible. }\end{array}$ \\
\hline $\begin{array}{l}\text { DOMAIN 2: } \\
\text { Physiological: } \\
\text { Complex }\end{array}$ & $\begin{array}{l}\text { CLASS N: } \\
\text { Tissue Perfusion } \\
\text { Management } \\
\end{array}$ & $\begin{array}{l}\text { Shock } \\
\text { management }\end{array}$ & 1. Monitor the hydration status, including weight, hourly diuresis and intake and output. \\
\hline DOMAIN 2: & CLASS N: & & 1. Monitor the weight. \\
\hline Physiological: & Tissue Perfusion & Fluid monitoring & 2. Monitor the intake and output. \\
\hline & Management & & 3. Keep an accurate record of the intake and output. \\
\hline $\begin{array}{l}\text { DOMAIN 2: } \\
\text { Physiological: } \\
\text { Complex }\end{array}$ & \begin{tabular}{|l} 
CLASS N: \\
Tissue Perfusion \\
Management \\
\end{tabular} & Shock prevention & 1. Control weight, intake and diuresis daily. \\
\hline & & & 1. Determine the patient's body expectations, according to the stage of development. \\
\hline & & & 2. Use a forward-looking guide in patient preparation for predictable body image changes. \\
\hline $\begin{array}{l}\text { DOMAIN 3: } \\
\text { Behavioral }\end{array}$ & $\begin{array}{l}\text { CLASS R: } \\
\text { Coping Assistance }\end{array}$ & $\begin{array}{l}\text { Body image } \\
\text { enhancement }\end{array}$ & $\begin{array}{l}\text { 3. Determine if the dislike perceived by certain physical characteristics creates dysfunctional } \\
\text { social paralysis in adolescents and other high-risk groups. }\end{array}$ \\
\hline & & & 4. Determine if there has been a recent physical change in the patient's body image. \\
\hline & & & $\begin{array}{l}\text { 5. Observe if there are affirmations that identify perceptions of body image that have to do with } \\
\text { shape and body weight. }\end{array}$ \\
\hline
\end{tabular}




\begin{tabular}{|l|l|l|l|}
\hline & & INTERVENCIONES & ACTIVIDADES \\
\hline & & & $\begin{array}{l}\text { 6. Use drawings of oneself as a mechanism for evaluating the perceptions of a child's body } \\
\text { image. }\end{array}$ \\
\cline { 3 - 4 } $\begin{array}{l}\text { DOMAIN 3: } \\
\text { Behavioral }\end{array}$ & $\begin{array}{l}\text { CLASS R: } \\
\text { Coping Assistance }\end{array}$ & $\begin{array}{l}\text { Body image } \\
\text { enhancement }\end{array}$ & $\begin{array}{l}\text { 7. Determine the perceptions of the patient and the family on changes in body image } \\
\text { relative to reality. }\end{array}$ \\
\cline { 3 - 4 } & & $\begin{array}{l}\text { 8. Help the patient at risk of suffering from anorexia or bulimia develop more realistic body } \\
\text { image expectations. }\end{array}$ \\
\hline
\end{tabular}

\section{DISCUSSION}

There are no previous studies that describe the application of anthropometric methods in the clinical practice of nursing. While we have identified several studies that relate methods of anthropometry and nutrition ${ }^{(12-16)}$ there is no evidence on the existence of studies in anthropometry and nursing care.

The standards of a professional nursing practice, developed by scientific associations and international regulatory bodies, include the different stages of the nursing process as requirements set in their nursing practice standards ${ }^{(17-18)}$. Likewise, in legislation both in Spain ${ }^{(19)}$ and at the international level(20), the nursing process is included as the main topic; in Spain, all the stages of the nursing process should be part of the minimum data included in the nursing care report.

To correctly apply the different stages of the nursing process, it is necessary to use various techniques and anthropometric measurements. If during the training process of nursing students the theoretical knowledge, skills and abilities related to anthropometry have not been acquired, errors can be made in the identification of the nursing diagnoses, interventions and activities to be developed, which would ultimately lead to providing inadequate care.

In the context of primary care, there is also agreement that many of the nursing diagnoses that nurses consider relevant require anthropometric measurements to identify the related factors or defining characteristics. In a study by Brito ${ }^{(21)}, 10$ of the 28 nursing diagnoses prioritized by a group of nurses in Spain required anthropometric techniques for their development: obesity (00232), overweight (00233), impaired skin integrity (00046), risk for falls (00155), impaired walking (00088), impaired physical mobility (00085), risk for injury (00035), sedentary lifestyle (00168), risk for overweight (00234) and fatigue (00093). Similarly, in a study by Besora et al. ${ }^{(22)}$, among the 36 nursing diagnoses prioritized by their frequency of use and relevance, there were seven that required anthropometric measurements. Additionally, in a study by Pérez et al. ${ }^{(4)}$, among the 10 most frequent nursing diagnoses in a health area, four required anthropometric measurements: impaired skin integrity (00046), imbalanced nutrition more than body requirements (00001), impaired tissue integrity (00044) and impaired physical mobility (00085).

Additionally, in the hospital environment, many authors have highlighted the need to use anthropometric measurements to identify various nursing diagnoses: imbalanced nutrition in patients with depression ${ }^{(23)}$; impaired physical mobility, risk for impaired skin integrity or imbalanced nutrition (less than body requirements) in institutionalized elderly patients ${ }^{(24)}$; imbalanced nutrition (less than body requirements), impaired skin integrity and impaired physical mobility in patients with chronic renal pathology $y^{(13,25)}$.
In relation to the anthropometric techniques and measurements required to perform nursing interventions, numerous studies have been identified, in both primary care and hospital care, where the need to use various anthropometric measurements to perform very common interventions in certain contexts were identified. In a study by Lucena et al. ${ }^{(26)}$, among the interventions that are commonly used in intensive care units, several are related, such as "exercise therapy: ambulation" or "pressure ulcer care" that require anthropometric measurements for their execution. Likewise, in the article by Scherb et al. ${ }^{(27)}$ on interventions in patients with heart failure, several interventions requiring anthropometric techniques were found ("fluid management", "nutrition management", among others). Finally, a study by Aguilar and Pancorbo-Hidalgo ${ }^{(28)}$ carried out in an elderly population also highlighted the use of many of the interventions that we have identified in our study: "self-care assistance: feeding", "exercise therapy: muscle control", "nutrition management" and "pressure ulcer care".

\section{Study limitations}

The comprehensive and independent review of the NANDA-I and NIC taxonomies should have been done by all members of the research except one member for review the possible existing discrepancies.

\section{Contributions to the fields of Nursing, Health or Public Policy}

These studies show that nursing diagnoses and interventions that require anthropometric techniques or measurements are commonly used. Therefore, to contribute to improving the diagnostic accuracy and correct execution of the care plans that include these interventions, it is necessary to ensure the correct education and training, both of the nursing students and the already graduated professionals, for the anthropometric techniques identified in our study. As we mentioned earlier, there are no previous studies that describe the application of anthropometric methods in the clinical practice of nursing.

\section{CONCLUSIONS}

This study identifies the main anthropometric techniques and measurements related to nursing diagnoses and interventions, included in the NANDA-I and NIC classifications, respectively.

Determining the necessary anthropometric techniques and measurements to be able to define a nursing diagnosis or execute nursing interventions in an appropriate manner can be very useful for their systematization.

In addition, it is necessary that nurses use these anthropometric techniques appropriately, to maintain the quality of the 
Application of anthropometric methods in the nursing process of nursing research Fernández SD, León SG, Bazán MJA, Cerro JLP, Marques-Vieira CMA, Rivas FJP.

measurement and avoid errors in the interpretation. It is therefore desirable that there be specific training in anthropometry on the part of nursing professionals that contributes to the proper determination of these measurements and improvement of the proper application of nursing care.

\section{FUNDING}

This work is funded by National Funds through the FCT Foundation for Science and Technology, I.P., within the scope of the project Refa UIDB/04279/2020.

\section{REFERENCES}

1. Alfaro-Lefevre R. Aplicación del proceso enfermero. Fomentar el cuidado en colaboración. 5a ed. Barcelona: Masson; 2003.

2. Clares JW, Guedes MV, Silva LF, Nóbrega MM, Freitas MC. Subset of nursing diagnoses for the elderly in Primary Health Care. Rev Esc Enferm USP. 2016; 50(2): 272-8. doi: 10.1590/S0080-623420160000200013

3. Herdman TH, Kamitsuru S. NANDA International nursing diagnoses: definitions and classification, 2015-2017. Oxford: Wiley-Blackwell; 2014.

4. Pérez-Rivas FJ, Santamaría-García JM, Minguet-Arenas C, Beamud-Lagos M, García-López M. Implementation and evaluation of the Nursing Process in Primary Health Care. Int J Nurs Knowl. 2012;23(1):18-28. doi: 10.1111/j.2047-3095.2011.01199.x.

5. Bulechek G, Butcher H, Dochterman J, Wagner C. Nursing interventions classification (NIC). 6th ed. St Louis, MO: Mosby; 2012.

6. Moorhead S, Johnson M, Mass M, Swanson E. Nursing Outcomes Classification (NOC). 5th ed. St Louis, MO: Mosby; 2012.

7. Pérez- Rivas FJ, Martín-Iglesias S, Pacheco-del Cerro JL, Minguet-Arenas C, García-López M, Beamud-Lagos M.. Effectiveness of nursing process use in primary care. Int J Nurs Knowl. 2016;27(1):43-8. doi: 10.1111/2047-3095.12073

8. Da Cruz CW, Bonfim D, Gaidzinski RR, Fugulin FM, Laus AM.. The use of Nursing Interventions Classification (NIC) in identifying the workload of nursing: an integrative review. Int J Nurs Knowl. 2014;25(3):154-60. doi: 10.1111/2047-3095.12031

9. Serrano MDM, Espinosa GM, Martínez CM. Anthropology of Nutrition. Techniques, methods and applications; 2003.

10. Van de Ven AH, Delbecq AL. The nominal group as a research instrument for exploratory health studies. Am J Public Health. 1972;62(3):33742. doi: 10.2105/ajph.62.3.337

11. Cantrill JA, Sibbald B, Buetow S. The Delphi and nominal group techniques in health services research. Int J Pharm Pract. 1996;4(2):67-74. doi: 10.1111/j.2042-7174.1996.tb00844.x

12. Díaz N, Meertens L, Solano L, Peña E. Nutritional characterization by anthropometrics of institutionalized and non-institutionalized elderly Venezuelan. Invest Clín. 2005;46(2):111-9.

13. Lorini C, Collini F, Castagnoli M, Di Bari M, Cavallini M C, Zaffarana N et al. Using alternative or direct anthropometric measurements to assess risk for malnutrition in nursing homes. Nutrition. 2014; 30(10):1171-6. doi: 10.1016/j.nut.2014.03.005

14. Stow R, Ives N, Smith C, Rick C, Rushton A. A cluster randomised feasibility trial evaluating nutritional interventions in the treatment of malnutrition in care home adult residents. Trials. 2015;16(1):433. doi: 10.1186/s13063-015-0952-2

15. Haney MO, Erdogan S. Factors related to dietary habits and body mass index among Turkish school children: a Cox's interaction modelbased study. J Adv Nurs. 2013;69(6):1346-56. doi: 10.1111/j.1365-2648.2012.06126.x

16. Wijnhoven HA, De Boer MR, Van Maanen MJ, Van Dongen DM, Kraaij SF, Smit T et al. Reproducibility of measurements of mid-upper arm circumference in older persons. J Hum Nutr Diet. 2013;26(1):24-31. doi: 10.1111/jhn.12010

17. American Nurses Association. Nursing Professional Development: Scope and Standards of Practice. Washington DC; 2010.

18. College of Registred Nurse of Nova Scotia. Standards for Nursing Practice; 2014.

19. Royal Decree 1093/2010, September 3, approving the minimum data set for clinical reports in the National Health System (BOE num. 225, 16 September 2010). Spanish.

20. Jarrín OF. Core elements of U.S. Nurse practice acts and incorporation of nursing diagnosis language. Int J Nurs Terminol Classif. 2010;21(4):166-76. doi:10.1111/j.1744-618X.2010.01162.x

21. Brito, PRB. Nursing diagnoses prioritized in primary care. Enferm Glob. 2009;(16):1-8. doi: 10.4321/S1695-61412009000200008

22. Besora I, Macià CDO, Llorach EG, Cariteu AO, Brichs CS. Standardized nursing diagnoses: guide for clinical practice in Primary Care. Metas de Enferm. 2008;11(3): 21-6.

23. Prokofieva M, Koukia E, Dikeos D. Mental Health Nursing in Greece: Nursing Diagnoses and Interventions in Major Depression. Issues Ment Health Nurs. 2016;37(8):556-62. doi: 10.3109/01612840.2016.1169466

24. Araújo DD, Carvalho RLR, Chianca. TM. Nursing diagnoses identified in records of hospitalized elderly. Invest Educ Enferm. 2014;32(2):225235. doi:10.17533/udea.iee.v32n2a05

25. Frazão CMFQ, Medeiros ABA, Paiva MDMN, Cruz Enders B, Oliveira Lopes MV, Brandão CL. Nursing diagnoses and adaptation problems among chronic renal patients. Invest Educ Enferm. 2015; 33(1): 119-27. doi:10.17533/udea.iee.v33n1a14

26. Lucena AF, Gutierrez MGR, Echer IC, Barros ALBL. Nursing interventions used in the clinical practice of an intensive care unit. Rev Latino-Am Enfermagem. 2018;18(5). doi: 10.1590/S0104-11692010000500006 
27. Scherb CA, Head BJ, Maas ML, Swanson EA, Moorhead S, Reed D, et al. Most frequent nursing diagnoses, nursing interventions, and nursing-sensitive patient outcomes of hospitalized older adults with heart failure: Part 1. Int J Nurs Terminol Classif. 2011;22(1):13-22. doi: 10.1111/j.1744-618X.2010.01164.X

28. Aguilar LV, Pancorbo-Hidalgo PL. Nursing diagnoses, outcomes and interventions identified in elderly patients with multiple pathologies after hospital discharge. Gerokomos. 2011;22(4):152-61. doi: 10.4321/S1134-928X2011000400002 